\title{
DW-ZCC Code Based on SAC-OCDMA Deploying Multi-Wavelength Laser Source for Wireless Optical Networks
}

\author{
Majid Moghaddasi • Saleh Seyedzadeh • Ivan \\ Glesk • Gandham Lakshminarayana • Siti \\ Barirah Ahmad Anas
}

Received: date / Accepted: date

\begin{abstract}
In this paper, double weight zero cross correlation (DW-ZCC) code is proposed for spectral amplitude coding optical code division multiple access (SAC-OCDMA) system. DW-ZCC takes the advantages of two previously proposed SAC-codes namely modified double weight (MDW) and zero-cross correlation (ZCC) codes, while providing optimized code length and maximum cross-correlation of zero. Although the proposed code can be utilized in SAC-OCDMA system with any optical medium, this research work focuses on outdoor wireless optical networks (WON) deploying multi-wavelength laser (MWL) source, where optical bandwidth is much limited. The mathematical and simulation analysis of proposed system employing direct decoding (DD) is developed, considering the influences of turbulence and system noises including relative intensity noise, optical beat interference $(\mathrm{OBI})$ and receiver noises. It is shown that employing DD detection; it is possible to completely avoid OBI which enhances system capability. The results show that SAC-OCDMAWON system is noticeably improved using DW-ZCC in term of transmission distance by at least $200 \mathrm{~m}$ in comparison with MDW and conventional ZCC codes.
\end{abstract}

Keywords Spectral Amplitude Coding - Wireless optical networks · Free space optics (FSO) · Multi-wavelength laser · Optical code division multiple access (OCDMA)

M. Moghaddasi, G. Lakshminarayanaa and S.B.A Anas

Wireless and Photonic Networks Research Center of Excellence (WiPNET), Department of Computer and Communication Systems Engineering, Faculty of Engineering, Universiti Putra Malaysia, 43400 UPM Serdang, Selangor, Malaysia

E-mail: majidmoghaddasi.photonics@gmail.com

S. Seyedzadeh and I. Glesk

Faculty of Engineering, University of Strathclyde, Glasgow G1 1XW, UK 


\section{Introduction}

Optical code division multiple access (OCDMA) system is considered as a promising solution to cater the growing demand for higher capacity in optical networks. It provides benefits such as security, quality of service (QoS) provision and flexibility in data rates [1-3]. Several encoding techniques have been implemented for OCDMA [4]. Spectral amplitude coding (SAC) among the encoding techniques has received great attention due to the ability to support asynchronous access, simplicity and lower costs $[5,6]$. In local and metropolitan area, wireless optical network is an attractive solution to transmit high bit rate data where installation of fiber cable is impossible (e.g. busy urban city or rural area with natural bars) [7]. Wireless optical network (WON) proposes a secure, license free and immune against electromagnetic interference transmission which makes it superior compared to radio frequency (RF) systems $[8,9]$.

Incorporation of OCDMA system with WON technology provide secure transmission and robustness against atmospheric adverse effects [10]. As wireless medium is much noisier than guided optical fiber, OCDMA system with spectral encoding scheme seems a proper choice compared to the temporal schemes [11]. Majority of SAC-OCDMA systems transmitting over optical fiber medium employ light emitting diode (LED) sources, however; they are not suitable light sources for WON transmission due to the low peak power and wide beam divergence. Hence, utilization of the multi-wavelength laser source offers better system performance for outdoor WON where there is high attenuation $[12,13]$. On the other hand, new developments in production of the low-cost multi-wavelength laser sources [14] and laser arrays such as high speed vertical-cavity surface-emitting laser (VCSEL) arrays, facilitate the practical utilization of multi-wavelength laser in industry $[15,16]$.

Development of SAC-OCDMA system employing complementary subtraction (CS) detection in WON was presented [17]. Generally, balanced receiver based detections such as CS are implemented for OCDMA codes with crosscorrelation of one and higher where there is overlap between signals of desired user and other users', hence; two additional destructive noises are generated namely multiple access interference (MAI) and optical beat interference (OBI) [18] in comparision with direct decoding (DD) that only detects nonoverlapping chips of desired user.

In this paper, a new code namely double weight zero cross correlation (DWZCC) is proposed. The proposed code offers cross correlation of zero between code-words, thereby, the performance of the proposed system implementing DD detection is derived. Mathematical and simulation models are developed to analyze the system performance of DW-ZCC code. The code is then compared with two well established codes namely modified double weight (MDW) [19] and zero-cross correlation (ZCC) [20]. The following sections describe the WON-OCDMA system description, code construction procedure, development of mathematical model and performance evaluation of DW-ZCC code. 


\section{SYSTEM DESIGN AND DESCRIPTION}

The schematic diagram of SAC-OCDMA in WOC that incorporates multiwavelength laser source is outlined in Fig.1. The utilized multi-wavelength source is illustrated based on experimental setup in [21]. In this setup, one tunable laser source (TLS) and one Raman pump (RPU) is utilized. Owing to nonlinear effects of Brillion scattering (BS) and Raman scattering (RS), the setup could produce multi-wavelength BrillouinRaman fiber laser (BRFL) with more than 90 stokes and $20 \mathrm{GHz}$ channel spacing in the linear cavity arrangement. Due to the adequate output uniformity over the channel spectra and high optical signal-to-noise ratio (OSNR), this multi-wavelength laser source can be utilized in wavelength division multiplexing (WDM) networks as well as OCDMA systems.

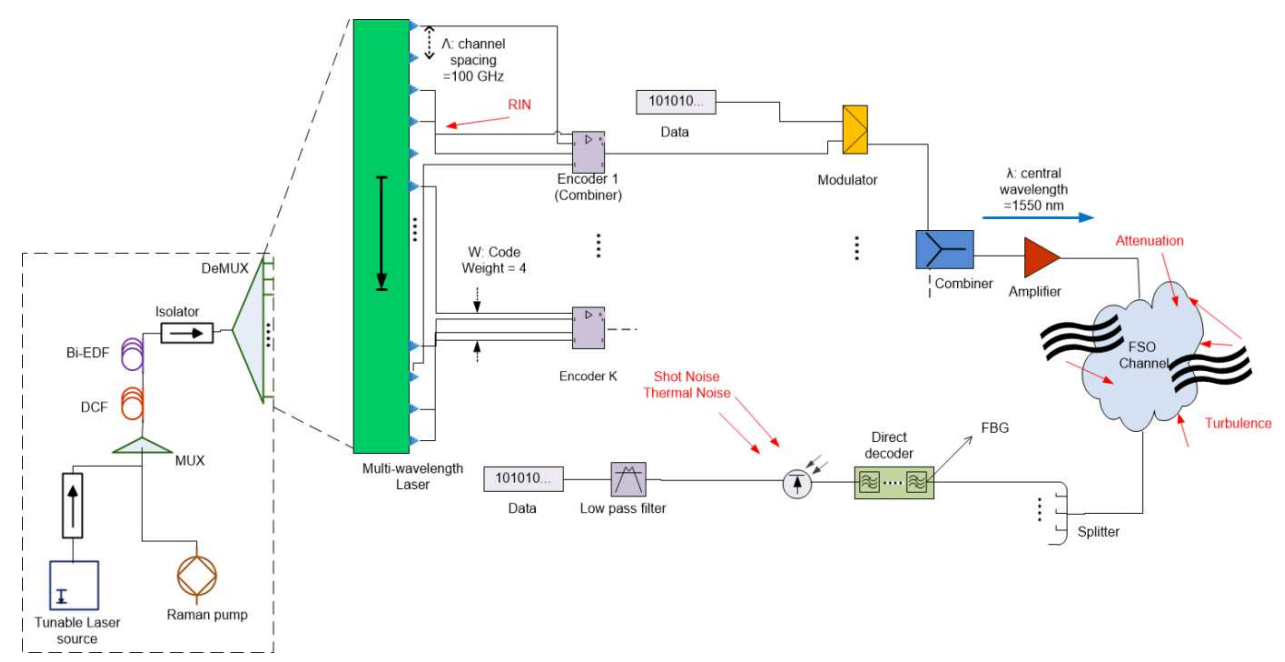

Fig. 1: Schematic of WON-OCDMA with SAC encoding and utilizing multiwavelength source in [21].

At the transmitter part, specific wavelengths corresponding to each code sequence are combined using an optical coupler and then modulated by a MachZehnder modulator. On-off Keying (OOK) modulation format with non-return to zero (NRZ) is considered for evaluation. The modulated signals from all users are then combined, amplified and transmitted through wireless medium. The purpose of using pre-amplification is to overcome dramatic attenuation incurred by air absorption and scattering. At the receiver, the combined optical signal is received using WON transceiver and then split among decoder where a DD decoder is applied exploiting a series of fiber Bragg gratings (FBGs). After forming the desired user's code signature, a photodetector (PIN) is used to convert optical signals to electrical form for bit error rate (BER) analysis. 


\section{DW-ZCC CODE}

\subsection{Code Properties}

DW-ZCC code takes the concept of two different codes MDW and ZCC and integrates the potential advantages of each. MDW code is constructed based on double weights and all chips appear in a pair, hence; the number of filters and subsequently system implementation cost is reduced [19]. On the other hand, in ZCC, there is zero cross correlation between all constructed code-words which results in totally suppression of MAI and increment of the system performance. In addition, DW-ZCC same as other ZCC codes uses DD detection, thereby the need for extra filters in lower arm of balanced receivers is eliminated which leads in further cost reduction [20]. The other advantage of DW-ZCC over conventional ZCC is simplicity in code construction as ZCC offers a recursive model for constructing codes in which generation of codes with weight of $\mathrm{W}$ requires the code signature with weight of $W-1$. Moreover, ZCC has restrictive condition of $K=W+1$; otherwise the system performance will suffer from longer code length that is not even calculable by a formula. Unlike ZCC, the proposed DW-ZCC supports flexible number of users and code weights which is more desirable for WON where a limited number of users are demanded. Furthermore, DW-ZCC provide code with shorter length than the code generated by ZCC, excluding the case where the number of users is equal to $W+1$ where the performance of the two codes are equal.

Table 1 demonstrates a comparison between MDW, ZCC and DW-ZCC codes in term of their code length $(N)$, and maximum cross-correlation $\left(R_{\max }\right)$ for generating code words with weight $W=6$, here $K$ represent the number of codewords or users. The evaluation in terms of mathematical and simulation analysis will be presented in subsequent sections. From Table 1, it can be seen that the code length of DW-ZCC is noticeable shorter comparing to ZCC. And although MDW generates code with shorter length than DW-ZCC, the $R_{\max }$ of MDW is equal one that leads to formation MAI and performance degradation.

\subsection{Code Construction}

The proposed DW-ZCC code is designated as $(N, W, \lambda)$ where $N$ and $W$ are the code length and code weight, respectively, and $\lambda=\sum_{i=1}^{N} a_{i} b_{i}=0$ denotes zero in-phase cross correlation between each pair of code-words, $A$ and $B$. Here $A=\left\{a_{1}, a_{2}, \cdots, a_{N}\right\}$ and $B=\left\{b_{1}, b_{2}, \cdots, b_{N}\right\}$ and $a_{i}$ and $b_{i}$ represent bit values. Therefore the matrix produces $K$ code sequences and $N$ represents as 
Table 1: COMPARISON BETWEEN MDW, ZCC \& DW-ZCC

(a) For the case $K>W+1$ where $K=10$

\begin{tabular}{rrrrr}
\hline \hline & $K$ & $W$ & $N$ & $R_{\max }$ \\
\hline MDW & 10 & 6 & 50 & 1 \\
ZCC & 10 & 6 & 71 & 0 \\
DW-ZCC & 10 & 6 & 60 & 0 \\
\hline
\end{tabular}

(b) For the case $K<W+1$ where $K=3$

\begin{tabular}{rrrrr}
\hline \hline & $K$ & $W$ & $N$ & $R_{\max }$ \\
\hline MDW & 3 & 4 & 9 & 1 \\
ZCC & 3 & 4 & 17 & 0 \\
DW-ZCC & 3 & 4 & 12 & 0 \\
\hline
\end{tabular}

$$
C=\left[\begin{array}{cccc}
C_{11} & C_{12} & \cdots & C_{1 N} \\
C_{21} & C_{22} & \cdots & C_{2 N} \\
\vdots & \vdots & \ddots & \vdots \\
C_{K 1} & C_{K 2} & \cdots & C_{K N}
\end{array}\right]_{K \times N}
$$

The length of generated code for $\mathrm{K}$ number of users with weight of $\mathrm{W}$ can be calculated as:

$$
N=K W
$$

Let $P(w)$ be the position of wth ' 1 ' in the code-word where $w=\{1,2, \cdots, W\}$, then the position of each pair of neighbor chips can be shown as $P^{\prime \prime}(i)$ and defined as

$$
\left.P^{\prime \prime}(i)\right)=P(i), P(i)+1
$$

and $i=1,2, \cdots, W / 2$, as the number of pair chips is half of the actual weight of the desired user. Equation (3) defines how pair chips are located in proportion to each other. For each user $\mathrm{k}$, $i$ th pair is defined as $P_{k}^{\prime \prime}(i)$ and can be calculated as

$$
P_{k}^{\prime \prime}(i)=\left\{\begin{array}{cr}
(k \times 2)-1,(k \times 2), & i=1 \\
P_{k}^{\prime \prime}(i-1)+[2(K-1)+2], & i>1
\end{array}\right.
$$

where $k=1,2, \ldots, K$.

Using equation (4), the $K \times N$ code matrix (equation (1)) is constructed with empty spaced between the 1's as chips.

The empty spaces between chips in each code-word will be filled by "0"s The number of zeros between each pairs can be calculated as:

$$
Z=2(K-1)
$$


The construction of proposed DW-ZCC code is only based on a mathematical recursive equation and unlike $\mathrm{MDW}$ and $\mathrm{ZCC}$ does not require step by step procedure and programming. Knowing the number of users, it is facile to construct the specific code-word for that user. This property becomes more useful in modification of en/decoders where there is no need to have the code matrix or previous code-words.

The following example presents code construction for 3 number of DWZCC code-words with weight 4 . The position of each pair chip is calculated by (4) for users 1 as $(1,2)$ and $(7,8)$, for user 2 as $(3,4)$ and $(9,10)$ and for users 3 as $(5,6)$ and $(11,12)$. Then the empty spaces is filled by " $0 \mathrm{~s}$. The code length which can be obtained using (2) is 12 and there are four 0s between each pair of chips. The constructed matrix is as following

$$
C=\left[\begin{array}{llllllllllll}
1 & 1 & 0 & 0 & 0 & 0 & 1 & 1 & 0 & 0 & 0 & 0 \\
0 & 0 & 1 & 1 & 0 & 0 & 0 & 0 & 1 & 1 & 0 & 0 \\
0 & 0 & 0 & 0 & 1 & 1 & 0 & 0 & 0 & 0 & 1 & 1
\end{array}\right]
$$

\section{THEORETIAL ANALYSYS WITH DIRECT DETECTION DECODING}

In order to simplify the analysis, the Gaussian approximation is used. In this case, $C_{k}(i)$ represents the $i$ th element of the $k$ th DW-ZCC code sequence. With regards to correlation at the photodetector, the code properties can be defined as:

$$
\sum_{i=1}^{N} C_{k}(i) C_{l}(i)=\left\{\begin{array}{cc}
W_{h}, & k=l \\
0, & k \neq l
\end{array}\right.
$$

where $W_{h}$ is the number of non-overlapped weights which are detected by photodetector.

To evaluate the system performance, the SNR of a back-to-back optical communication system is obtained as [17]

$$
S N R=\frac{\langle I\rangle^{2}}{\left\langle I^{2}\right\rangle}=\frac{I_{b}^{2}}{i_{n b}^{2}}
$$

where $i_{n b}^{2}$ is the power of noise sources which exist in the photocurrent and $I_{b}$ is the average desired received photocurrent at the detector and can be calculated by [17]

$$
I_{b}=R b W_{h} P_{r}
$$

where $b \in\{1,0\}$ is the bit value which presents the transmission of ' 1 ' or ' 0 ' by desired user. $P_{r}$ is received power per chip and $R$ represent photodiode responsivity. $W_{h}$ is the number of chips for each user which are absorbed by photodetector and its value is dependent on detection method as well as coding technique. For complementary subtraction decoding $W_{h}=W$ which means all 
chips of a user are detected by photodetector. However in DD detection which is the used method in this research work, the value of $W_{h}$ is dependent on the code type. For instance, this value is $W_{h}=w / 2$ for MDW code and $W_{h}=1$ for modified quadratic congruence (MQC) code.

The variance of total noise power, $i_{n b}^{2}$ for an OCDMA system with a multiwavelength laser source can be defined as: [17]

$$
i_{n b}^{2}=i_{O B I b}^{2}+i_{R I N b}^{2}+i_{s h b}^{2}+i_{t h}^{2}
$$

Where $i_{O B I b}^{2}, i_{R I N b}^{2}, i_{s h b}^{2}$ and $i_{t h}^{2}$ are the variance of noises related to optical beat interference (OBI), relative intensity noise (RIN), shot noise, and thermal noise, respectively. It should be noted that OBI occurs only because of beating between desired and interfering pulses as well as between interfering pulses themselves, on the same photodetector [22,23]. In DD detection, only desired pulses hit the photodetector, therefore; OBI is avoided in this technique $\left(i_{O B I b}^{2}=0\right)$. OBI is a dominant noise in SAC-OCDMA with multi-wavelength laser source [13], and so subpressing this noise will result in significant performance improvement. Shot noise is induced by hit pulses related to desire user, $W_{k}$, at the decoder and can be defined as

$$
i_{s h b}^{2}=2 E\left(b W_{h} P_{r}+x P_{c}\right) B_{e}
$$

Where $x$ is the event of the whole interfering pulses from the possible interfering users out of $K-1$ that transmit bit ' 1 '. $P_{c}$ is the optical power of the crosstalk pulses and for DD detection is equal to 0 . Then

$$
i_{s h b}^{2}=2 E b W_{h} P_{r} B_{e}
$$

$B_{e}$ is electrical bandwidth and $E$ is the electron's charge. Assuming that the number of interfering pulses that are sending bit ' 1 ' at every chips for all users are equal, the average value for $x$ will be [13]

$$
x=\frac{W_{h}^{2}(K-1)}{2 N}
$$

On the other hand, RIN occurs at transmitter of the desired signal and all other interfering users cause crosstalk with desired user signal. Therefore $W_{h}=W$ and

$$
i_{R I N b}^{2}=R I N\left(b W P_{r}+x P_{c}\right)^{2} B_{e}
$$

where RIN is the noise factor with a typical value between -130 to -160 $\mathrm{dBHz}^{-1}[24]$. Thermal noise can be attained by:

$$
i_{t h}^{2}=\frac{4 K_{B} T B_{e}}{R_{L}}
$$

where $T$ is temperature, $K_{B}$ is the Boltzmann constant, and $R_{L}$ is the load resistor. Then the total noise power variance can be written as 


$$
i_{n}^{2}=i_{R I N}^{2}+i_{s h}^{2}+i_{t h}^{2}
$$

The detection of the bits is done by comparing the total signal current with threshold current $I_{D}$.

Then the BER of the optical signal transmitted in the additive white Gaussian noise (AWGN) channel indicated by $B E R_{0}(I)$ and can be achieved by:

$$
B E R_{0}(I)=\frac{1}{2}\left[Q\left(\frac{I_{1}-I_{D}}{i_{n 1}}\right)+Q\left(\frac{I_{D}-I_{0}}{i_{n 0}}\right)\right]
$$

Where $I_{0}$ and $I_{1}$ are the total signal current for $I_{b}(b=0$ or 1$), i_{n 0}$ and $i_{n 1}$ are noise power of transmitting zero and one, respectively. $Q$ function is

$$
Q(x)=\frac{1}{2 \pi} \int_{x}^{\infty} \exp \left(-\frac{y^{2}}{2}\right) d y
$$

then considering the case that $I_{D}$ is optimum.

$$
I_{D}=\frac{i_{n 0} I_{1}+i_{n 1} I_{0}}{i_{n 0}+i_{n 1}}
$$

the total probability of error can be summarized as [22]

$$
\operatorname{BER}_{0}(I)=Q\left(\frac{I_{1}-I_{0}}{i_{n 1}+i_{n 0}}\right)
$$

On the other hand $P_{r}$ in a WON-CDMA system with SAC, can be calculated by $[17]$

$$
P_{r}=\frac{P_{t} D^{2} e^{-\Omega L}}{N(\theta L)^{2}}
$$

here, $P_{t}$ is the total transmitted power into the WON channel, $D$ is the receiver aperture diameter, $\Omega$ is the attenuation coefficient, $N$ is code length, and $L$ is the transmission distance while the $\theta$ represents the beam divergence.

To evaluate the performance of the whole system, BER is calculated. Hence, the impact of the turbulence must be considered. The strength of the turbulence is presented by Rytov variance and for plane wave propagation is

$$
\sigma_{R}^{2}=1.23 C_{n}^{2} K_{\text {own }}^{7 / 6} L^{11 / 6}
$$

where $C_{n}^{2}$ is the refractive index structure coefficient and $K_{\text {own }}=2 \pi / \lambda$ is the optical wave number.

Atmospheric turbulence is a stochastic phenomenon which can be analyzed by statistical methods. The reliability and behavior of the system is determined by the probability density function (PDF) of the randomly fading irradiance. Here, Gamma-Gamma distribution is chosen due to potentiality in describing behavior of weak to strong turbulences. The Gamma-Gamma PDF of the terms of normalized irradiance I is given by [25] 


$$
P_{G}(I)=\frac{2(\alpha \beta)^{\frac{(\alpha+\beta)}{2}}}{\Gamma(\alpha) \Gamma(\beta)} I^{\left[\frac{(\alpha+\beta)}{2}-1\right]} K_{\alpha-\beta}(2 \sqrt{\alpha \beta I})
$$

where $\Gamma($.$) is the Gamma function and K_{(\alpha-\beta)}$ is the modified Bessel function of the second kind of order $\alpha-\beta$. Here $\alpha$ and $\beta$ are the effective number of small and large-scale eddies of scattering environment and defined as

$$
\alpha=\left\{\exp \left[\frac{0.49 \sigma^{2}}{\left(1+1.11 \sigma_{R}^{\frac{12}{5}}\right)^{\frac{7}{6}}}\right]-1\right\}^{-1}
$$

and

$$
\beta=\left\{\exp \left[\frac{0.51 \sigma^{2}}{\left(1+0.69 \sigma_{R}^{\frac{12}{5}}\right)^{\frac{5}{6}}}\right]-1\right\}^{-1}
$$

The BER of an optical signal which has been transmitted in the GammaGammadistributed channel can be presented as [26]

$$
B E R=\int_{0}^{\infty} B E R_{0}(I) P_{G}(I) d I
$$

BER in the aforementioned equation is used to determine the performance of the proposed system.

\section{SOFTWARE SIMULATION DESIGN}

Fig. 2 shows similar system implemented using Optisystem version 10. A multiwavelength laser module with linewidth of $20 \mathrm{MHz}$ and output power of $3 \mathrm{dBm}$ is used is used where the number of users is set 3 WDM MUX with insertion loss 1.5. RIN noise factor was considered $137 \mathrm{~dB} / \mathrm{Hz}-1$ for both simulation and mathematical approach. A random bit generator and electrical pulse generator is used to modulate optical signal utilizing a MZM modulator. Also a combiner with $5.4 \mathrm{dBm}$ insertion loss and $0.1 \mathrm{dBm}$ excess loss is used to combine all 3 users signals.

Then the superimposed signal is amplified with an EDFA with average gain up to $28 \mathrm{~dB}$ and noise figure of $5 \mathrm{~dB}$ which reinforces the input signal to $20 \mathrm{dBm}$. The EDFA is used as power booster to provide adequate input power to the free space medium before high loss is experienced.

At the receiver side, similar setup with Fig. 1 is implemented where the signal is decoded by a series of FBGs corresponding to the wavelengths encoded at transmitter. The FBGs have reflectivity of $99 \%$ and $0.5 \mathrm{~dB}$ loss. Finally, the optical signal is converted into electrical signal using PIN photodetector The PIN has responsivity of $1 \mathrm{~A} / \mathrm{W}$ and thermal noise of 16e-024 W/Hz.Shot 


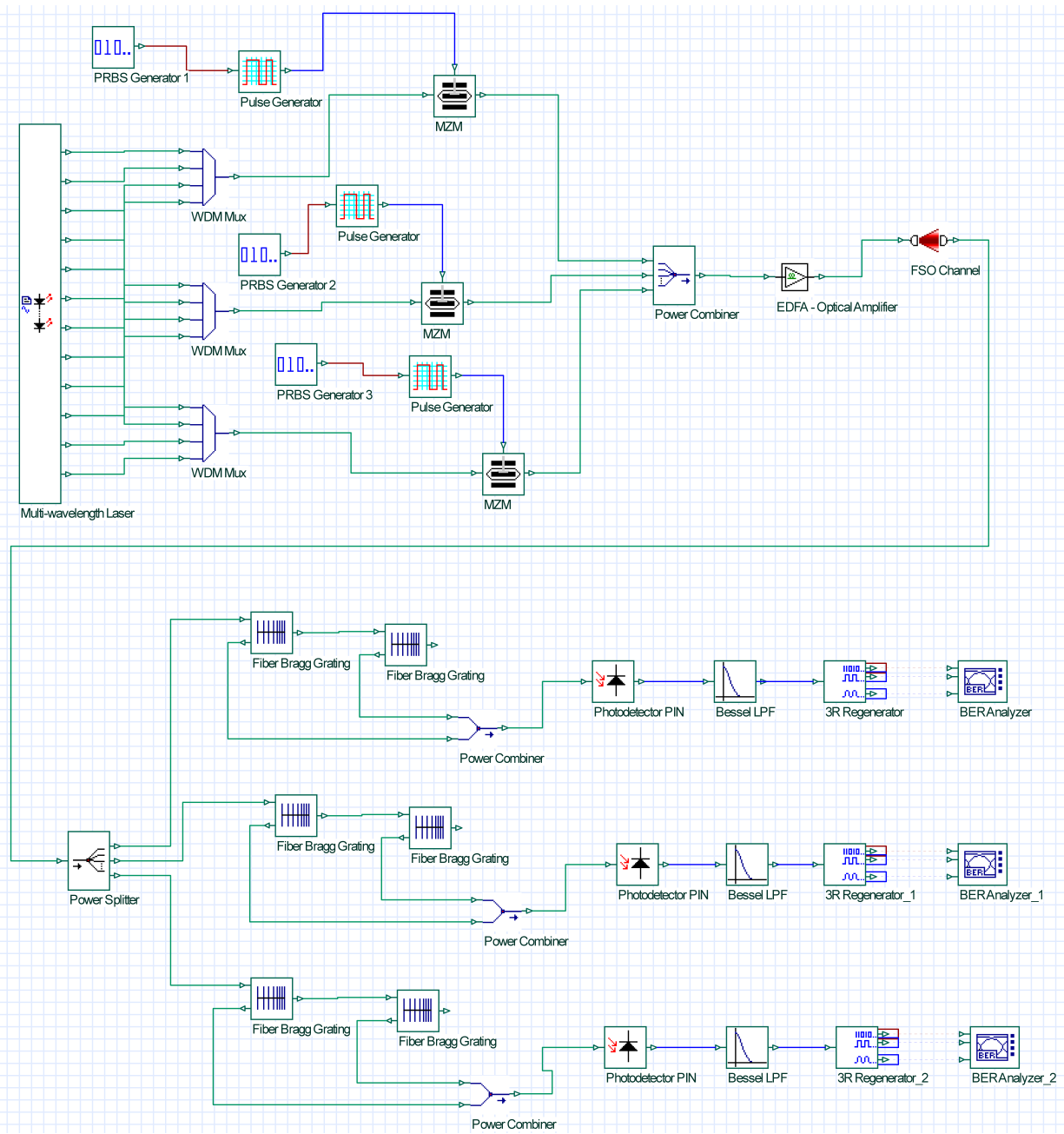

Fig. 2: Simulation setup for SAC in Wireless Optical Networks.

noise value is calculated by simulator software based on other parameters values. The electrical signal is then passed from a four order low-pass Bessel filter with cut-off frequency of $1.8 \mathrm{GHz}$ to recover primary data.

\section{THEORETICAL AND SIMULATION RESULTS}

The parameters used in the analysis are chosen to provide a fair comparison with other published works and listed in Table 2. WON-OCMDA system employing MDW, ZCC and DW-ZZ with 10 number of subscribers each with code 
Table 2: Typical parameters used in the analysis

\begin{tabular}{lll}
\hline \hline Symbol & Parameter & Value \\
\hline$\theta$ & Beam divergence & $0.5 \mathrm{mrad}$ \\
$\lambda$ & Operating wavelength & $1550 \mathrm{~nm}$ \\
$D$ & Receiver aperture diameter & $8 \mathrm{~cm}$ \\
$B_{e}$ & Electrical bandwidth & $2.5 \mathrm{GHz}$ \\
$\sigma_{R}^{2}$ & Rytov variance & 1 \\
$\Omega$ & Weather attenuation & $3 \mathrm{~dB} / \mathrm{km}$ \\
$P_{c}$ & Crosstalk power & $-30 \mathrm{dBm}$ \\
$P_{t}$ & Total transmitted power & $20 \mathrm{dBm}$ \\
\hline \hline
\end{tabular}

weight of 8 is investigated. The moderate turbulence condition is considered for this particular experiment and transmission distance is set to $1.7 \mathrm{~km}$.

In order to calculate the BER of each configuration 10 simulations are performed and the worst case is considered as final performance. Each user operates at data rate of $2.5 \mathrm{~Gb} / \mathrm{s}$ and the pseudo-random bit sequence length is set to $2^{13}-1$.

Figure 3 illustrates the effect of simultaneous number of users on the performance of the system. It can be seen that DW-ZCC outperform both codes supporting 11 and 21 more number of users comparing ZCC and MDW, respectively, reference to the BER of $10^{-9}$. MDW shows the worst performance due to the fact that the number of overlapping chips is higher than others which results in decreased received power and subsequently SNR. The advantage of lower length of proposed DW-ZCC code over conventional ZCC is obviously indicated with this results. Reduced code length leads in receiving higher $P_{r}$ (refer to Eq. (21)) which eventuates increased $I_{b}$ and SNR (refer to Eq. (8 \& $9))$.

In Fig. 4, the influence of turbulence on the system performance is evaluated where DW-ZCC is used for spectral coding with 10 numbers of users and weight of 6 . The Rytov variance $\sigma_{R}^{2}$ of $0.4,1$ and 2 represents weak, moderate and strong turbulences, respectively. Reference to BER of $10^{-9}$, the transmission distance for weak, moderate and strong turbulences is 1.95, 2.15 and 2.45 $\mathrm{km}$, respectively.

In Fig. 5 performance of the system against transmission distance increment for three codes, MDW, ZCC and DW-ZCC, is evaluated with number of users 3 and weight 4 using simulation and mathematical analysis. Simulation setup is implemented using OptiSystem software version 10 as a proof for reliability of proposed mathematical model. It can be observed that both analysis results prove that DW-ZCC is able to provide the best performance in transmission up to $3.2 \mathrm{~km}$. This range is suitable for last mile access network which is a significant bottleneck for most of the service providers. As seen in Fig. 5 (a) and (b) both ZCC and DW-ZCC code outperform MDW and 


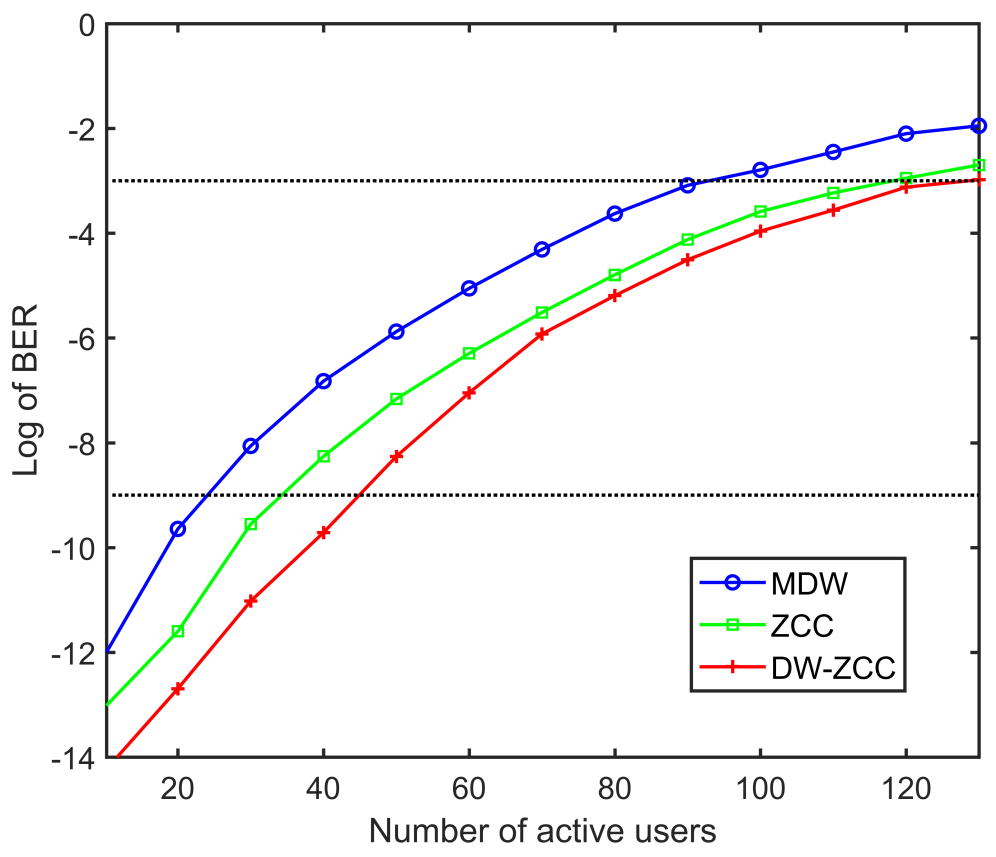

Fig. 3: Log of BER vs number of simultaneous users.

DW-ZCC shows $200 \mathrm{~m}$ improvement in term of transmission distance over its conventional counterpart.

Fig. 6 illustrates eye diagram for MDW, ZCC and DW-ZCC for $2 \mathrm{~km}$ transmission distance. It can be observed that eye diagram for DW-ZCC is better than others in terms of eye height and jitter. With measurement of the jitter, the values (the length between points $\mathrm{A}$ and $\mathrm{B}$ in figure 6 ) were observed $0.25,0.171$ and 0.152 bit period for MDW, ZCC and DW-ZCC respectively. This means less jitter for DW-ZCC and so better performance for it.

\section{Conclusion}

In this paper, a new code family namely $\mathrm{VW}-\mathrm{ZCC}$ was proposed for WONOCDMA system deploying SAC technique. The nature of the proposed code is in a way to perfectly eliminate the effect of OBI which is known as the significant performance degradation factor in system employing multi-wavelength lasers as optical sources, such as WON. The design and code construction of VW-ZCC was explained in detail and a mathematical model was developed for performance analysis and comparison of proposed code with other well-known SAC-cods namely MDW and conventional ZCC. In developing numerical model, influential noises in transmitter, receiver and channel including 


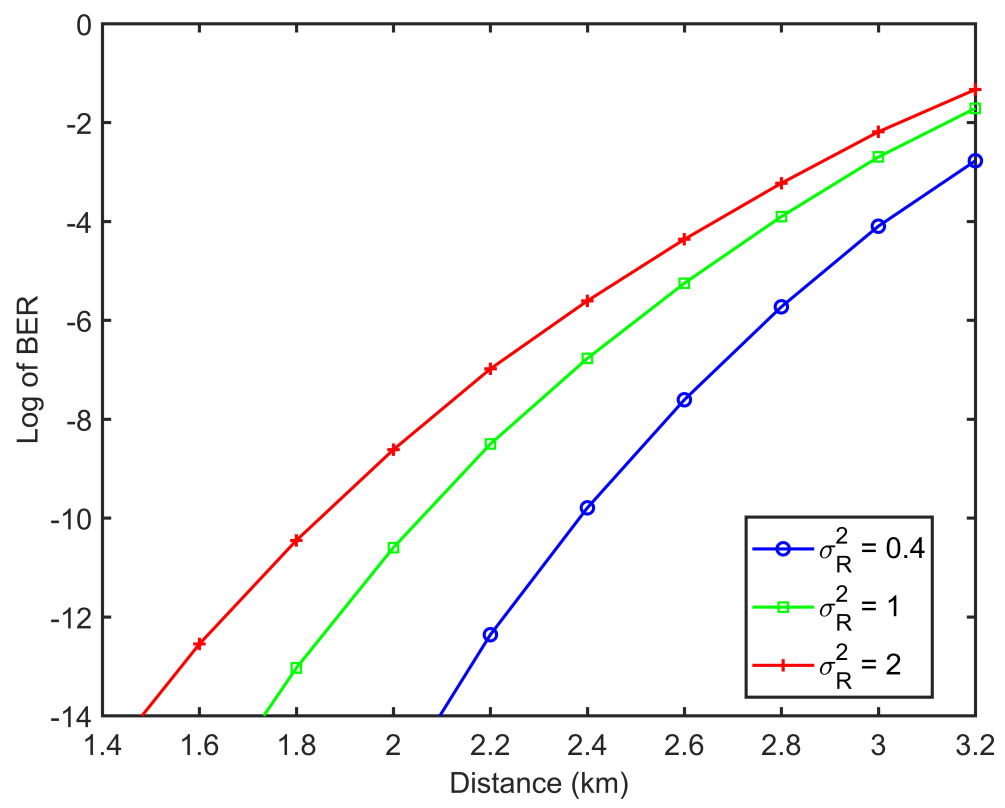

Fig. 4: Log of BER against transmission distance with DW-ZCC for different turbulence values.

thermal noise, shot noise, RIN, channel attenuation and also turbulence were taken into account. Results demonstrated that OCDMA system with VW-ZCC noticeably surpass both MDW in which the overlapping chips are higher than new code and conventional ZCC which offers an increased code length with same weight. The performance of VW-ZCC in different turbulence strength was presented and number of supportable users in normal situation was calculated using mathematical model. In support of numerical model, a simulation setup was developed and results was discussed. Both simulation and mathematical results reveals that proposed VW-ZCC is a promising code and can be implemented in OCDMA based WON to provide desired performance.

\section{References}

1. A. Taiwo, M. Moghaddasi, D. Kuje, Y. Idriss, M. Mokhtar, IET Optoelectronics 10(6), $227(2016)$

2. S. Seyedzadeh, G.A. Mahdiraji, R.K.Z. Sahbudin, A.F. Abas, S.B.A. Anas, Optical Fiber Technology 20(5), 495 (2014)

3. Y.K. Huang, I. Glesk, C.M. Greiner, D. Iazkov, T.W. Mossberg, T. Wang, P.R. Prucnal, Optics express 15(12), 7327 (2007)

4. M. Moghaddasi, S. Seyedzadeh, S.B. Ahmad Anas, in 2014 IEEE REGION 10 SYMPOSIUM (IEEE, 2014), pp. 181-184. DOI 10.1109/TENCONSpring.2014.6863021. URL http://ieeexplore.ieee.org/lpdocs/epic03/wrapper.htm?arnumber $=6863021$ 


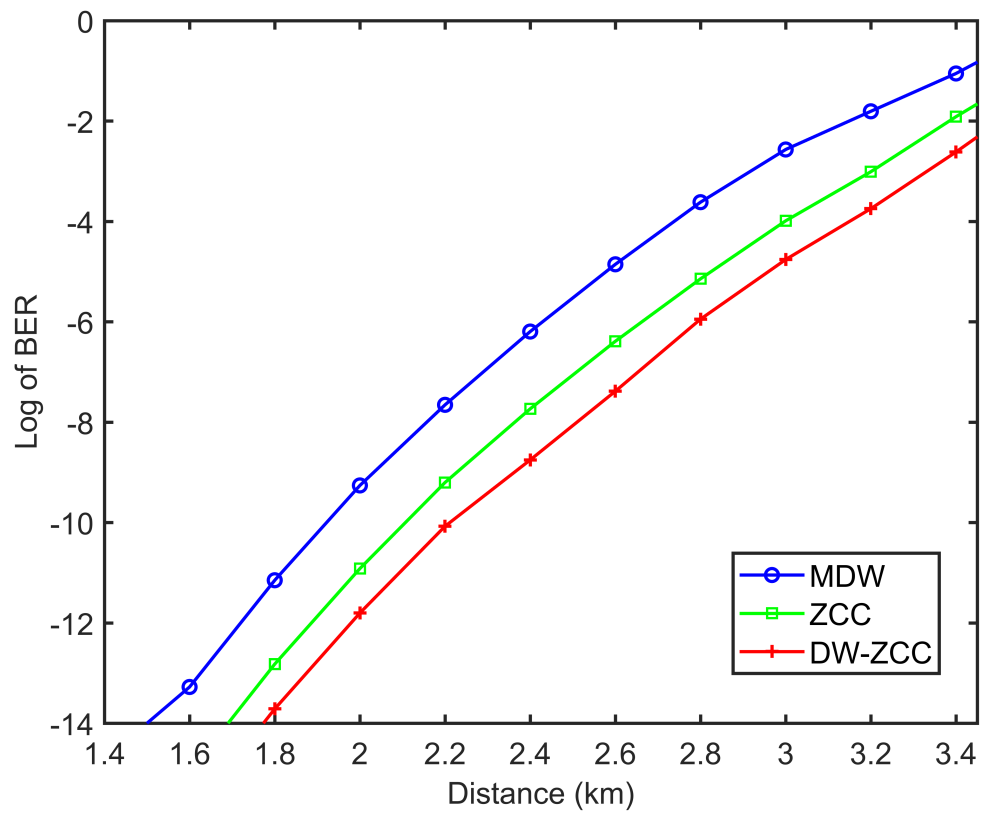

(a)

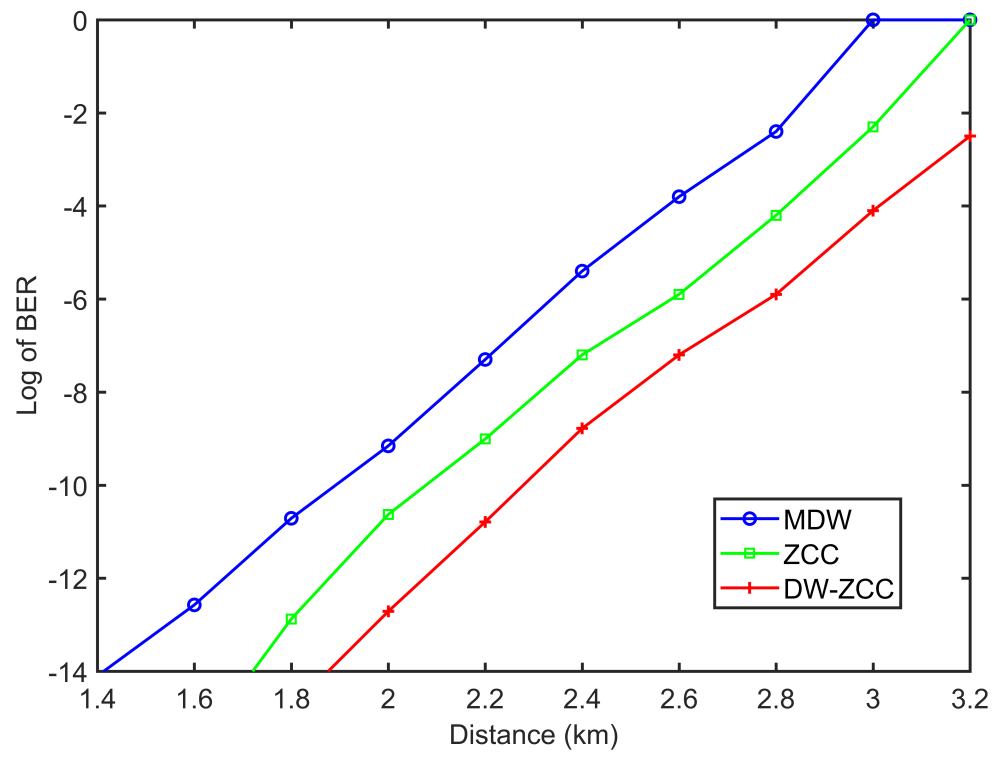

(b)

Fig. 5: BER vs. transmission distance for (a) analytical result (b) simulation result for moderate turbulence 


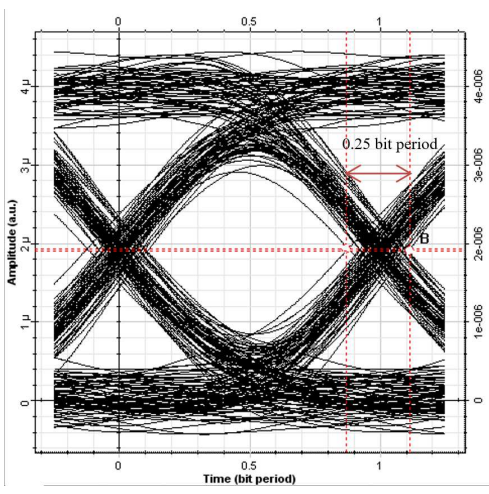

(a)

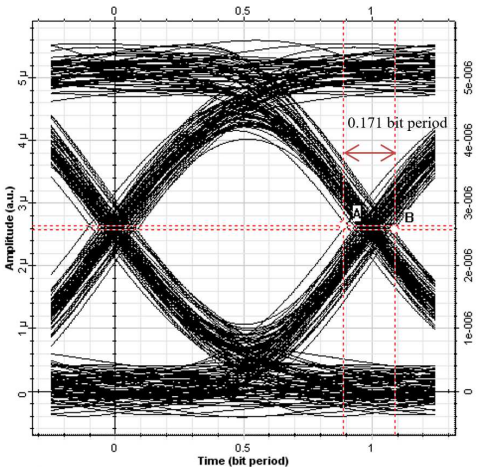

(b)

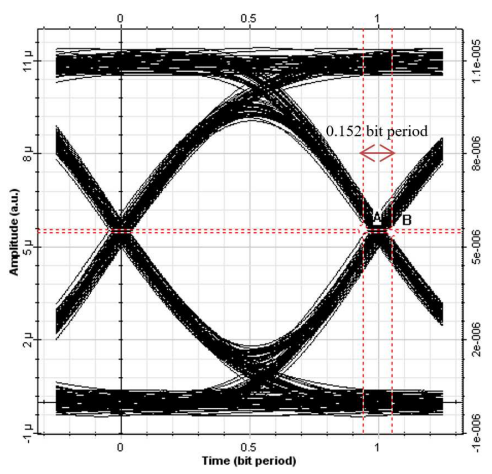

(c)

Fig. 6: Eye diagram for (a) MDW, (b) ZCC and (c) DW-ZCC showing produced jitter for each code.

5. S. Seyedzadeh, M. Moghaddasi, S.B.A. Anas, Journal of Telecommunications and Information Technology 3, 50 (2016)

6. H. Chen, S. Xiao, L. Yi, J. Shi, P. Yang, Y. Dong, W. Hu, Photonics Technology Letters, IEEE 24(5), 335 (2012)

7. N. Cvijetic, S.G. Wilson, M. Brandt-Pearce, Photonics Technology Letters, IEEE 19(2), $103(2007)$

8. E. Bayaki, D.S. Michalopoulos, R. Schober, Communications, IEEE Transactions on 60(12), $3797(2012)$

9. J. Perez, S. Zvanovec, Z. Ghassemlooy, W.O. Popoola, Optics express 22(3), 3208 (2014)

10. H. Yin, D.J. Richardson, chap 1, 36 (2008)

11. H. Ghafouri-Shiraz, M.M. Karbassian, Optical CDMA Networks: Principles, Analysis and Applications (Wiley-IEEE Press, 2012)

12. Z. Wei, H. Shalaby, H. Ghafouri-Shiraz, Journal of Lightwave Technology 19(9), 1274 (2001)

13. M. Moghaddasi, G. Mamdoohi, A.S.M. Noor, M.A. Mahdi, S.B.A. Anas, Lasers in Engineering 33(4), 247 (2016)

14. Z. Wang, H. Wu, M. Fan, Y. Li, Y. Gong, Y. Rao, Optics express 21(24), 29358 (2013)

15. A. Pruijmboom, LASER FOCUS WORLD 50(12), 19 (2014) 
16. V. Karagodsky, B. Pesala, C. Chase, W. Hofmann, F. Koyama, C.J. Chang-Hasnain, Optics express 18(2), 694 (2010)

17. M. Moghaddasi, G. Mamdoohi, A.S.M. Noor, M.A. Mahdi, S.B.A. Anas, Optics Communications 356, $282(2015)$

18. K.J. Dexter, D.A. Reid, L.P. Barry, Photonics Technology Letters, IEEE 21(22), 1662 (2009)

19. S. Aljunid, M. Ismail, A. Ramli, B. Ali, M. Abdullah, IEEE Photonics Technology Letters 16(10), 2383 (2004). DOI 10.1109/LPT.2004.833859

20. M.S. Anuar, S.A. Aljunid, N.M. Saad, S.M. Hamzah, Optics Communications 14(14), 2659 (2009). DOI 10.1016/j.optcom.2009.03.079

21. G. Mamdoohi, A.R. Sarmani, A.F. Abas, M.H. Yaacob, M. Mokhtar, M.A. Mahdi, Optics Express 21(16), 18724 (2013)

22. N.T. Dang, A.T. Pham, Optics express 18(10), 9922 (2010)

23. T.M. Bazan, D. Harle, I. Andonovic, Lightwave Technology, Journal of 24(10), 3583 (2006)

24. J.M. Senior, M.Y. Jamro, Optical fiber communications: principles and practice (Pearson Education, 2009)

25. G.K. Rodrigues, V.G.A. Carneiro, A.R. da Cruz, M.T.M.R. Giraldi, Optics Communications 305, 42 (2013)

26. Z. Wang, W.D. Zhong, S. Fu, C. Lin, Photonics Journal, IEEE 1(6), 277 (2009) 